Article

\title{
TeamMate: a longitudinal study of health in working farm dogs. III. Factors affecting the risk of death, euthanasia or re- tirement from work.
}

\author{
Katja E. Isaksen ${ }^{1, *}$, Lori Linney ${ }^{2}$, Helen Williamson ${ }^{2}$, Elizabeth J. Norman ${ }^{3}$, Nick J. Cave ${ }^{1}$ and Naomi Cogger ${ }^{1}$ \\ 1 School of Veterinary Science, Massey University, Palmerston North 4410, New Zealand \\ 2 Vetlife, Timaru, New Zealand \\ 3 College of Sciences, Massey University, Palmerston North 4410, New Zealand \\ * Correspondence: katjaisaksen01@gmail.com
}

Simple Summary: Working farm dogs are essential to many livestock farmers, but little is known about what increases their risk of being lost from the workforce through dying, being euthanized or being retired. A study carried out in New Zealand found that the majority of farm dogs that were lost from work during a four-year period died or were euthanized rather than being retired, and owners reported that acute injuries or illnesses were the most common cause. However, $65 \%$ of dogs that died or were retired were at least seven and 38\% at least ten years old, showing that working farm dogs often work into their old age. Data from physical examinations done by veterinarians showed that lameness almost doubled dogs' risk of being lost from work, independently of their age. Our results show that further research into what causes lameness in working farm dogs, and how this lameness can be avoided, could make a significant positive impact on the health and welfare of these dogs.

\begin{abstract}
Working farm dogs are essential to many livestock farmers. Little is known about factors that influence dogs' risk of being lost from work. This paper explores risk factors for farm dogs being lost through death, euthanasia and retirement. All enrolled dogs were working and minimum 18 months old. Five data collection rounds were done over four years. Data about dogs were collected from owners and dogs were given physical examinations by veterinarians. Dogs that were lost from work were counted and owner-reported reasons for loss were recorded. Multivariable logistic regression modelling was used to investigate risk factors for loss. Of 589 dogs, 81 were lost from work. Of these, 59 dogs died or were euthanized and 22 were retired. Farm dogs tended to reach high ages, with $38 \%$ being 10 years or older when last examined. Acute injury or illness was the most commonly owner-reported reason for loss. Age group $(\mathrm{P}<0.0001)$ and lameness $(\mathrm{P}=0.04$, $\mathrm{OR}=1.8$ ) significantly affected dogs' risk being lost. These results expand our knowledge about factors that affect health, welfare and work in farm dogs. Further investigation into reasons for lameness may help improve health and welfare in working farm dogs.
\end{abstract}

Keywords: risk factors, longevity, death, euthanasia, retirement, longitudinal, TeamMate, working dogs, herding dogs, working farm dogs

\section{Introduction}

There are a range of concerns around the health and welfare of working farm dogs. Previous studies have found that traumatic injury and musculoskeletal injury and disease are common in farm dogs in New Zealand [1-5]. Further, owners report concern that as many as $19 \%$ of their dogs may be underweight [3]. Supporting this, around one third of dogs enrolled in the TeamMate study could be considered as underweight if assessed using body condition scoring [4]. However, it is not known how specific conditions or whether having low body condition scores affect the health or welfare of farm dogs. 
In addition to affecting welfare, it is plausible that factors related to health affect the risk of farm dogs being lost from the working population. Working farm dogs are an essential part of livestock farming in New Zealand and in other parts of the world [6,7]. The loss of dogs from work can be disruptive to the effective running of farms and put extra pressure on farmers and their remaining dogs. Knowing which factors are likely to increase dogs' risk of death or retirement can help dog owners and veterinarians mitigate those risks to ensure that dogs have the longest and healthiest working lives possible. Such knowledge may also help inform further research into how the identified risk factors might be avoided. For example, in addition to being commonly recorded in working farm dogs, musculoskeletal injury and disease have been reported as common causes of euthanasia and death in police, military and guide dogs [8-10]. However, while cross-sectional studies have been carried out into reported reasons for dogs being lost from work, studies that analyze longitudinal data to investigate which factors might put dogs at increased risk of death or retirement are rare. Such risk factor analysis can reveal exposures that make dogs more susceptible to developing the conditions that cause them to be removed from work. Due to this lack of investigation there may be important risk factors that are currently being overlooked by researchers and veterinarians.

The aims of this study were to fill this gap in knowledge by investigating risk factors that influenced death, euthanasia or retirement of dogs during the course of the TeamMate study. Additionally, owner-reported reasons for death or retirement were reported and compared with the significant risk factors revealed by our analysis. Determining whether specific factors related to demographics, husbandry and health are associated with the risk of working farm dogs dying, being euthanized or being retired will help researchers, veterinarians and dog owners decide which areas to focus on to improve dogs' care and husbandry.

\section{Methods}

\subsection{Study design}

TeamMate is a longitudinal study focusing on working farm dogs on the South Island of New Zealand. The study design and data collection procedure are presented in detail in a previous publication [4]. To summarize, a total of $126 \mathrm{dog}$ owners associated with 116 farms participated in the study and 641 working farm dogs were enrolled. All working farm dogs belonging to participating owners were included if they were least 18 months old and working with livestock regularly.

Data collection was begun in May 2014. Data was collected approximately every eight to nine months subsequently, and data from five data collection rounds were included in the current study. The fifth data collection round was completed in November 2017. Figure 1 is a flowchart showing the start dates for each data collection round and how many dogs, owners, and farming properties were enrolled at each round. At each data collection round, farm dog owners were visited on the farm where they worked, new dog owners and dogs were enrolled, and data was collected. New dog owners and dogs were enrolled up to and including the third data collection round. New dogs included dogs belonging to previously enrolled owners that had been acquired or had become old enough to be enrolled in the study between farm visits. Some new properties were registered subsequently to the third data collection round due to participating dog owners moving or changing jobs.

At each farm visit, including on enrolment, all enrolled dogs were physically examined by veterinarians, and dog owners were interviewed to collect information about the dogs' husbandry, feeding, and work. Scribes were responsible for filling in the questionnaires and taking note of any clinical findings. The physical examination included manipulation of all the major limb joints and encouraging the dogs to trot for a short distance to check for lameness. All physical abnormalities were recorded, irrespective of their clinical significance. The questionnaires that were used as part of the study are available as supplementary materials to a companion research article [4]. 
All veterinarians and scribes were trained to ensure data collection was performed in a standardized way, with veterinarians asked to record specific clinical signs rather than diagnoses. Training included a run-through of all questionnaires and how they should be completed, as well as practical sessions that involved filling in the questionnaires and examining, scoring, and measuring farm dogs. During training sessions, normal ranges of motion of the joints were demonstrated in healthy working farm dogs.

Abnormalities noted on physical examination were systematically categorized. Categories were not mutually exclusive, and dogs could have multiple recorded abnormalities, also in the same anatomical location. Categorization was carried out by a single veterinarian (LL) and checked by another person with training in animal health. Categories that were unclear or incomplete were rechecked by a veterinarian (LL and/or NJC). The complete system used to categorize physical abnormalities is available as supplementary materials to a companion research article [4].

In the current study, we included all observations of dogs where no data was missing from the relevant explanatory variables and information was available on whether dogs died or were retired subsequently to the observation. To avoid excessive reduction of our sample size, potential explanatory variables were not included in the multivariable analysis if more than five percent of observations did not have a recorded value for the variable. See Figure 2 for details on how many observations were excluded and the reasons they were excluded. 


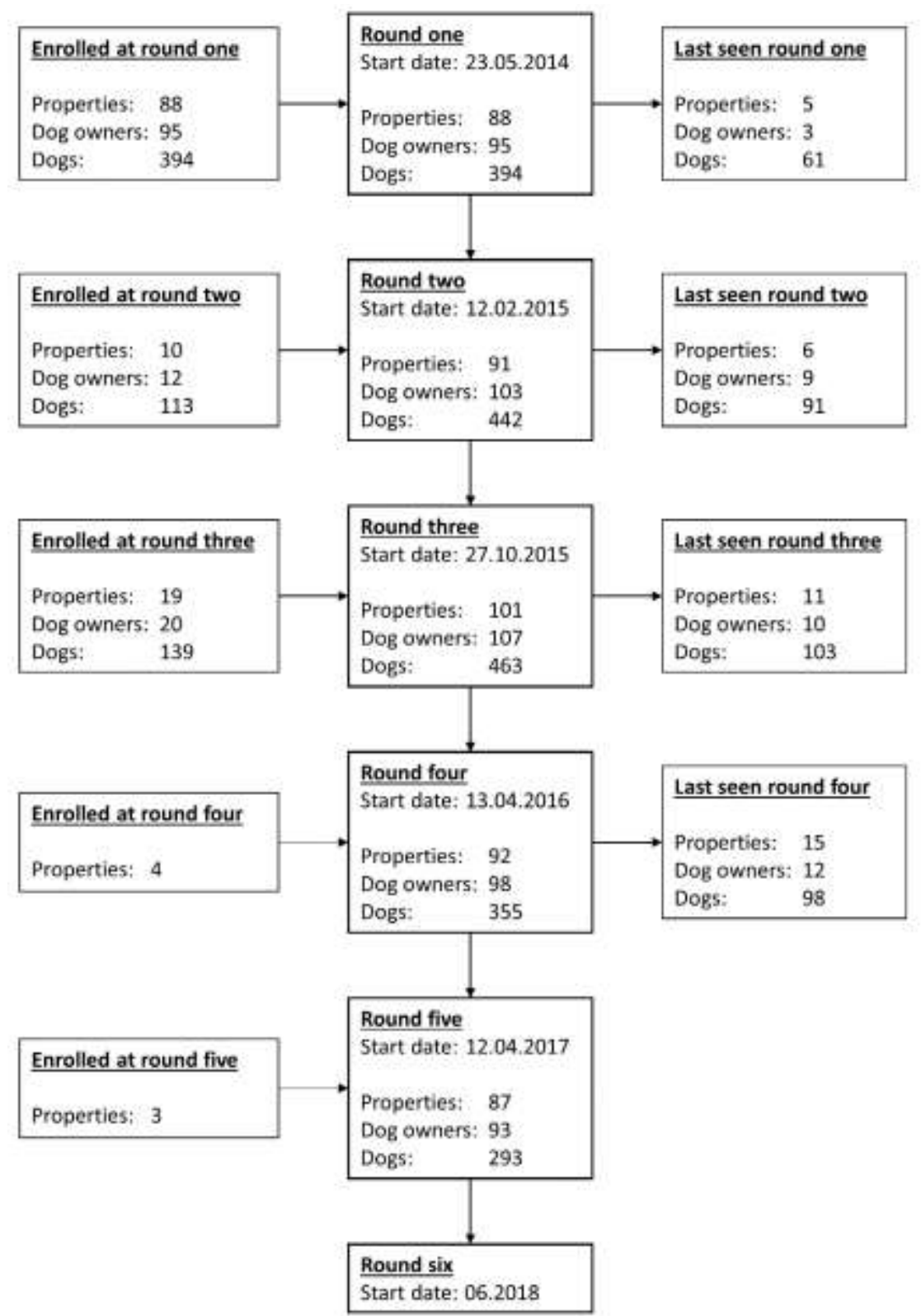

Figure 1: Flowchart showing the start dates of each data collection round as well as the number of farms, dog owners and dogs enrolled in TeamMate up to and including the fifth round of farm visits. Additionally, 14 properties, 16 dog owners and 68 dogs missed at least one round of data collection. Note that data for the sixth data collection round was not yet available at the time of writing. This figure was previously published by the authors [4] and is licensed for re-use under the Creative Commons Attribution 4.0 International licence. 


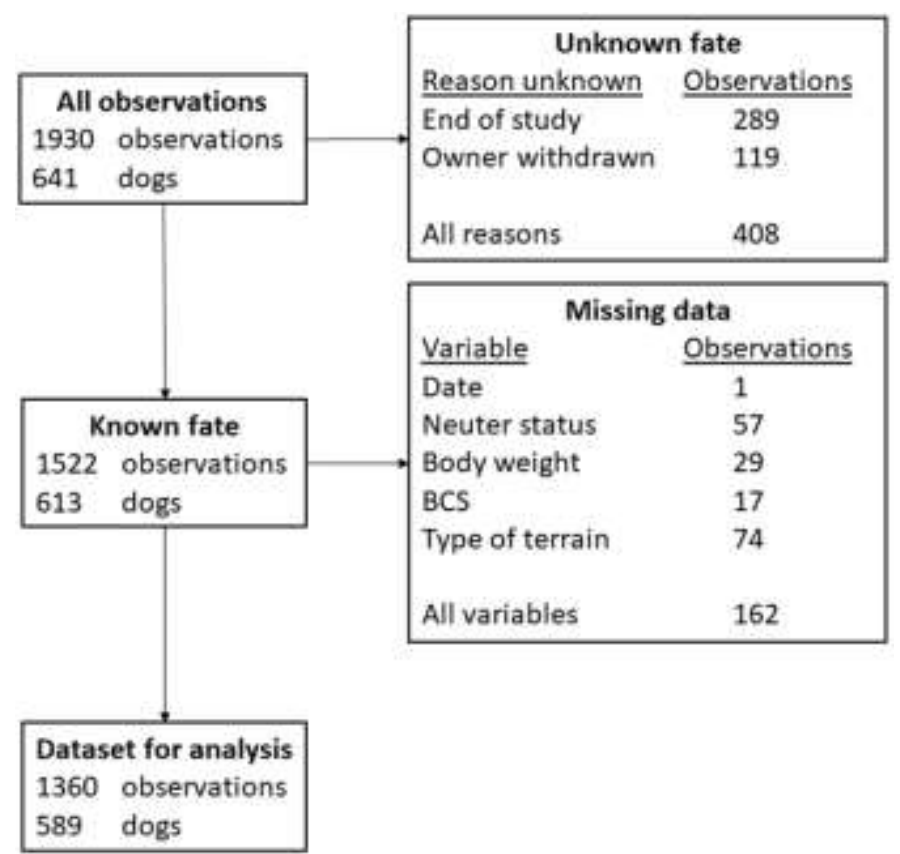

Figure 2: Flowchart showing the number of observations that were removed from the analysis due to missing information. As observations could have missing data in more than one variable, the sum of observations with missing data in the different variables do not equal the total number of observations that were removed. Note that dogs that had one or more observations removed could still be present in the dataset.

\subsection{Outcomes - absence, death and retirement of dogs}

The outcome variable analyzed in this study was whether or not dogs were lost from the workforce through death or retirement. At each farm visit following their enrolment to the study, dogs were classified as present or absent. Dogs that were still working on the property but not available for physical examination on the day of data collection were classified as being present but not examined. The fates of dogs that were present on the last farm visit made to the owner were recorded as 'working with original owner'. Absent dogs were classified as having been lost from the workforce if the owner reported them as having died or having been retired from work for any reason. Absent dogs that were not dead or retired were not classified as having been lost from the workforce, and their fates were categorized as 'rehomed', 'sold', 'loaned' or 'withdrawn from the study'. Dogs reported as loaned included both dogs that had been loaned out to a different owner and dogs that had been returned to their owner after being loaned. Dogs were occasionally reported as having been retired to a smaller farm. These dogs were assumed to still be working, although in a reduced capacity, and were recorded as having been rehomed rather than being lost from the workforce. Where possible, the reason why a dog was absent was recorded. No data was available on whether health events or conditions that were reported by the owner as being the cause of a dog being absent, had been confirmed or diagnosed by a veterinarian.

Data were analyzed to assess the risk of the dog dying or being retired following each farm visit and physical examination. Observations where no information was available regarding the further fates of dogs were excluded from analysis. These observations were either the last before a dog owner withdrew from the study or recorded during the final round of data collection (Figure 2).

\subsection{Explanatory variables}

Variables that were considered to be potential risk factors relating to dogs dying or being retired were screened for inclusion in the analysis. These variables are listed in Table 1. Clinical abnormalities were grouped according to their overall type - mainly whether the abnormality affected a specific body system - and were included in the 
analysis if they were present in $10 \%$ of dogs or more on enrolment to the TeamMate study [4]. Lameness can be caused by musculoskeletal injury or disease, but also by other conditions such as footpad abrasions or nail injuries. Similarly, not all the clinical abnormalities that were included in the musculoskeletal category cause lameness. Therefore, despite often being associated with musculoskeletal conditions, lameness was analyzed as a separate risk factor to musculoskeletal conditions.

Observations conducted on each dog were numbered, with enrolment being Observation 1 , and each following observation being numbered sequentially. These observation numbers were included in the analysis to account for the progression of time during the course of the study.

Dog types were classified as 'Heading dog', 'Huntaway' or 'other' based on information provided by the dog owner. The types of work dogs were reported to carry out were classified as 'heading', 'hunting', 'yard work' or combinations of these. More details on the different types of working farm dogs found in New Zealand, how dogs enrolled in TeamMate were classified, their average body weights, which types of work they were recorded to do, and how these types of work were classified can be found in an earlier publication [4].

Body condition was scored using a nine-point numeric scale where 1 is severely underweight, 9 is morbidly obese, and 4 to 5 is considered ideal [11]. Body condition in relation to dogs' lean body mass was quantified by calculating the ratio of the predicted lean mass to skeletal size using a novel equation developed by measuring lean mass and body size in 20 working farm dogs [12].

Dog owners were asked to report the ages of dogs on the enrolment of the dogs and on follow-up observations. At $10 \%$ of observations subsequent to enrolment dogs' ages were not recorded. In these cases, the dog's age was calculated based on the dog's reported age at enrolment and the time passed.

Dogs that were enrolled in TeamMate and had information available relating to whether or not they had died or been retired following at least one observation were eligible to be included in the current study. Data from observations were excluded from multivariable analysis if they contained missing observations in any of the variables that were examined as potential risk factors.

Table 1: List of explanatory variables that were assessed as possible risk factors for the death or retirement of working farm dogs.

\begin{tabular}{cc}
\hline Type & Variable names \\
\hline Observation & Number of observations including enrolment. \\
\hline Characteristics of dogs & $\begin{array}{c}\text { Age, sex, neuter status, type of dog, body weight, } \\
\text { body condition score (1-9), ratio of predicted lean } \\
\text { body mass to skeletal size. }\end{array}$ \\
\hline $\begin{array}{c}\text { Findings on physical } \\
\text { examination }\end{array}$ & $\begin{array}{c}\text { Number of recorded abnormalities, presence of } \\
\text { lameness on trot, presence of musculoskeletal } \\
\text { abnormalities, presence of skin abnormalities, } \\
\text { presence of mouth and teeth abnormalities, presence } \\
\text { of eye abnormalities, presence of reproductive } \\
\text { abnormalities. }\end{array}$ \\
\hline Work related variables & Type of work, number of days worked in week \\
& preceding observation. \\
\hline Other & Type of terrain on property, presence of bedding in \\
& kennel.
\end{tabular}


The number of enrolled dogs were counted stratified by their fate at the conclusion of the study. Dogs that died or were retired were counted and stratified by the reported reason for death or retirement and their age group on their last observation in the study. Percentages and 95\% CIs were calculated for all stratified counts.

The risk of a dog dying, being euthanized or being retired after each farm visit was analyzed using univariable and multivariable binary logistic regression models. Odds ratios were calculated by exponentiating the model beta-coefficients. All models were checked for significance using P-values derived from log-likelihood ratio tests (LRT). Potential risk factors were included in the multivariable analysis if they had a recorded value in at least $95 \%$ of dog observations, and the significance of the log-likelihood ratio test was less than $P<0.2$ during univariable screening. The best-fit multivariable model was developed using backwards single-term deletion, where all potential risk factors were included in the first model tested and the variable with the smallest association with the risk of death or retirement was removed at each step. Backward elimination continued until all variables had a $P$-value for the log-likelihood ratio test of less than 0.05 , which was considered to be statistically significant. The observation numbers for each dog were retained in all multivariable models, irrespective of effect size or significance, in order to account for the passing of time from the first to the last data collection round. Pairwise interactions were tested for all variables in the final multivariable model. To account for repeated measures over the course of the study, individual dog and dog owner identification numbers were added to the final multivariable model as nested random effects. The change in model fit caused by adding the random effects was tested using a log-likelihood ratio test.

All continuous explanatory variables were checked for linearity. The log-odds probabilities of dogs being lost were plotted against each continuous variable using a smoothed (loess) line [13]. The resulting plot was visually examined for linearity. Additionally, a quadratic term was added to the univariable model to allow the regression line to follow a curved path [13]. The quadratic term was created by centering and squaring the values of the variable. Centering was done to avoid collinearity with the original predictor. The assumption of linearity was checked by examining whether the quadratic term was significantly associated with whether dogs died or were retired. If the $P$-value extracted from a log-likelihood ratio test was smaller than 0.05 and the smoothed line of the log-odds probabilities had a clear curvature, the assumption of linearity was determined to have been broken. In such cases the explanatory variable was converted to a categorical, removing the assumption of linearity from the model.

To evaluate the quality of fit of the final multivariable model we examined the area under the receiver operator characteristic (ROC) curve. Additionally, the residuals generated by the mixed logistic regression model were checked for outliers that might indicate problems with model fit.

All calculations and data analysis were done using $R$ version 4.0.x [14]. The values necessary to plot loess smoothed lines for checking linearity of continuous predictors were generated using the loess() function in the car package [15] and the logit() function in the stats package [14]. Random effects models were fitted using the lme4 package [16]. The receiver-operator curve was generated and plotted using the pROC package [17]. The residuals of the final multivariable mixed model were plotted using the qqnorm() and qqline() functions in the stats package.

\section{Results}

In total, 1930 observations were recorded from the 641 dogs that were enrolled in TeamMate. Four hundred ninety-three observations were removed from the dataset due to a lack of information about the fate of the dog following the relevant observation or due to missing data in variables that were examined as risk factors for death or retirement. Figure 2 shows how many observations were removed from the final dataset and which explanatory variables contained missing values. Full sets of data with no missing 
observations in the relevant variables were available for 1360 observations of 589 working farm dogs belonging to 120 dog owners. Table 2 shows the distribution of dogs by sex, age group at enrolment and type.

\subsection{Fates of dogs and reasons for death or retirement}

In total, 81 of 589 dogs had the outcome of interest, that is they were lost from the workforce through dying, being euthanized or being retired during the study period. Table 3 lists the fates of all 589 dogs following the last observations that were included in this study, Table 4 shows the owner-reported reasons why dogs died, were euthanized or were retired, and Table 5 shows the age groups of dogs that died or were retired.

Table 2: Population data relating to 589 working farm dogs that were enrolled in TeamMate and included in the risk factor analysis.

\begin{tabular}{cccc}
\hline \multicolumn{2}{c}{ Variables } & Number of dogs & Percentage \\
\hline \multirow{2}{*}{ Sex } & Female & 269 & $46 \%$ \\
& Male & 320 & $54 \%$ \\
\hline \multirow{4}{*}{ Age on enrolment } & 1.5 to 2.9 years & 179 & $30 \%$ \\
& 3 to 4.9 years & 164 & $28 \%$ \\
& 5 to 6.9 years & 104 & $18 \%$ \\
& 7 to 9.9 years & 107 & $18 \%$ \\
& 10 years and above & 35 & $6 \%$ \\
\hline \multirow{3}{*}{ Type of dog } & Heading dog & 282 & $48 \%$ \\
& Huntaway & 288 & $49 \%$ \\
& Other & 19 & $3 \%$ \\
\hline
\end{tabular}

Table 3: The fates of 589 working farm dogs enrolled in TeamMate.

\begin{tabular}{cccc}
\hline Fate of dog & Number of dogs & \multicolumn{2}{c}{$\mathbf{\% ( 9 5 \% ~ C I ) ~}$} \\
\hline Working with original owner & 427 & 72 & $(69-76)$ \\
Dead or euthanised & 59 & 10 & $(8-12)$ \\
Retired from work & 22 & 4 & $(2-5)$ \\
Rehomed & 32 & 5 & $(4-7)$ \\
Sold & 44 & 7 & $(5-10)$ \\
Loaned & 4 & 1 & $(0-1)$ \\
Not reported & 1 & 0 & $(0-1)$ \\
\hline
\end{tabular}

Table 4: Owner-reported reasons for death or retirement of 81 dogs enrolled in TeamMate.

\begin{tabular}{|c|c|c|c|c|c|c|c|}
\hline \multirow[b]{2}{*}{ Reported reason } & \multicolumn{2}{|c|}{$\begin{array}{c}\text { Died or euthanised } \\
n=59\end{array}$} & \multicolumn{3}{|c|}{$\begin{array}{c}\text { Retired } \\
\mathrm{n}=22\end{array}$} & \multicolumn{2}{|c|}{$\begin{array}{l}\text { All dead or retired } \\
\qquad \mathrm{n}=81\end{array}$} \\
\hline & Dogs & $\%(95 \% \mathrm{CI})$ & Dogs & & $95 \% \mathrm{CI})$ & Dogs & $\%(95 \% \mathrm{CI})$ \\
\hline Acute injury or illness & 21 & $36(23-48)$ & 1 & 5 & $(0-13)$ & 22 & $27(17-37)$ \\
\hline Old age & 6 & $10 \quad(2-18)$ & 4 & 18 & $(2-34)$ & 10 & $12(5-20)$ \\
\hline Chronic injury or illness & 8 & $14 \quad(5-22)$ & 1 & 5 & $(0-13)$ & 9 & $11 \quad(4-18)$ \\
\hline Sudden death & 8 & $14 \quad(5-22)$ & - & - & - & 8 & $10 \quad(3-16)$ \\
\hline Behaviour & 6 & $10 \quad(2-18)$ & 0 & 0 & & 6 & $7 \quad(2-13)$ \\
\hline Not reported & 10 & 17 & 16 & 73 & & 26 & 32 \\
\hline
\end{tabular}


Table 5: Age on last observation of 81 dogs enrolled in TeamMate that were reported as having died or been retired from work.

\begin{tabular}{|c|c|c|c|c|c|c|c|c|}
\hline \multirow{3}{*}{$\frac{\text { Age on last observation }}{1.5 \text { to } 2.9 \text { years }}$} & \multicolumn{2}{|c|}{$\begin{array}{c}\text { Died or euthanised } \\
n=59\end{array}$} & \multicolumn{3}{|c|}{$\begin{array}{c}\text { Retired } \\
\mathrm{n}=22\end{array}$} & \multicolumn{3}{|c|}{$\begin{array}{l}\text { All dead or retired } \\
\qquad \mathrm{n}=81\end{array}$} \\
\hline & \multirow{2}{*}{$\begin{array}{c}\text { Dogs } \\
10\end{array}$} & $\%(95 \% \mathrm{CI})$ & \multirow{2}{*}{$\frac{\text { Dogs }}{1}$} & \multicolumn{2}{|c|}{$\%(95 \% \mathrm{CI})$} & \multirow{2}{*}{$\begin{array}{c}\text { Dogs } \\
11\end{array}$} & \multicolumn{2}{|c|}{$\%(95 \% \mathrm{CI})$} \\
\hline & & $17 \quad(7-27)$ & & 5 & $(0-13)$ & & 14 & $(6-21)$ \\
\hline 3 to 4.9 years & 6 & $10 \quad(2-18)$ & 0 & 0 & & 6 & 7 & $(2-13)$ \\
\hline 5 to 6.9 years & 10 & $17 \quad(7-27)$ & 1 & 5 & $(0-13)$ & 11 & 14 & $(5-22)$ \\
\hline 7 to 9.9 years & 18 & $31(19-42)$ & 4 & 18 & $(2-34)$ & 22 & 27 & $(17-37)$ \\
\hline 10 years and older & 15 & $25(14-37)$ & 16 & 73 & $(54-91)$ & 31 & 38 & $(28-49)$ \\
\hline
\end{tabular}

3.2. Analysis of risk factors for death or retirement

Dogs' ages, body weights, body condition scores and ratio of predicted lean mass to skeletal size were found to have non-linear relationships with the log-odds of dogs dying, being euthanized or being retired. These potential explanatory variables were therefore converted to categorical values before analysis. Dogs' ages were categorized using the age groups used in a previous publication [4]. Dogs body weights were divided into quartiles. Body condition scores were categorized according to whether they are considered underweight $(1-3)$, ideal $(4-5)$ or overweight $(6-9)$ according to the World Small Animal Veterinary Association [11]. The ratio of predicted lean mass to skeletal size was divided into quartiles.

Table 6 shows an overview of potential explanatory variables that were excluded from univariable screening due to having recorded values in less than $95 \%$ of the 1522 observations where dogs had a known fate. If these four variables had been included in the multivariable analysis the sample size would have been reduced by 662 observations of 349 dogs.

Univariable screening for significance was done using the final dataset of 1360 observations of 589 dogs. Table 7 shows an overview of potential explanatory variables that were excluded after univariable screening due to being insufficiently associated with the risk of dogs dying, being euthanized or being retired from work $(P(L R T)>0.2)$. The results of univariable testing of potential explanatory variables that were included in the multivariable analysis and model building are listed in Table 8.

Table 9 presents the odds ratios calculated from the best-fit multivariable logistic mixed model, and the change in model fit when each of the remaining explanatory variables were removed. Dogs in the youngest and oldest age groups had the highest risk of dying, being euthanized or being retired, with dogs between three and 4.9 years having the lowest risk. Dogs were twice as likely to die or be retired if they were recorded as being lame on trot. Additionally, the presence of eye abnormalities had an effect on the risk of dogs dying, being retired or being euthanized (Odds ratio $=1.9,95 \% \mathrm{CI}=1.0-3.6$, $\mathrm{P}(\mathrm{LRT})=0.06)$ after accounting for age group and the presence of lameness. While this effect did not meet the threshold for significance and was removed from the final multivariable model, the effect of eye abnormalities had a relatively much stronger effect than any other tested variables is therefore mentioned here.

The final multivariable mixed model had an area under the ROC curve of 0.76 (95\% $\mathrm{CI}=0.71-0.82)$.

Table 6: Number and percentage of observations that had a recorded value for the respective variable. $P$-values were derived from log-likelihood ratio tests of univariable logistic regression models examining the association between potential explanatory variables and the risk of observations being followed by dogs dying or being retired. The listed explanatory variables were not included in univariable screening or multivariable analysis as they had recorded values in less than $95 \%$ of observations. Data is from 1522 observations of 613 dogs that were enrolled in the TeamMate project. 


\begin{tabular}{|c|c|c|c|c|c|}
\hline \multirow[b]{2}{*}{ Variable name } & \multirow[b]{2}{*}{ Variable levels } & \multicolumn{3}{|c|}{ Observations } & \multirow[b]{2}{*}{$P($ LRT $)$} \\
\hline & & $\mathbf{n}$ & $\%$ & Died or retired & \\
\hline $\begin{array}{l}\text { Number of days worked } \\
\text { week before observation }\end{array}$ & (Count value) & 1421 & 93 & 99 & $<0.001$ \\
\hline \multirow{4}{*}{$\begin{array}{c}\text { Ratio of predicted lean } \\
\text { body mass to skeletal size } \\
\text { (quartiles) }\end{array}$} & Below 3.4 & 244 & \multirow{4}{*}{69} & 21 & \multirow{4}{*}{0.47} \\
\hline & 3.5 to 3.7 & 257 & & 13 & \\
\hline & 3.8 to 4.1 & 271 & & 19 & \\
\hline & 4.2 and higher & 272 & & 19 & \\
\hline \multirow{2}{*}{$\begin{array}{c}\text { Presence of bedding in } \\
\text { kennel }\end{array}$} & No & 735 & \multirow{2}{*}{90} & 56 & \multirow{2}{*}{0.47} \\
\hline & Yes & 636 & & 42 & \\
\hline \multirow{6}{*}{ Types of work } & Heading only & 571 & \multirow{6}{*}{94} & 42 & \multirow{6}{*}{0.35} \\
\hline & Hunting only & 59 & & 7 & \\
\hline & Heading and yard work & 102 & & 8 & \\
\hline & Hunting and yard work & 401 & & 27 & \\
\hline & Heading, hunting and yard work & 235 & & 16 & \\
\hline & Other combinations & 58 & & 1 & \\
\hline
\end{tabular}


Table 7: The results of univariable screening of potential explanatory variables for the risk of observations being followed by dogs dying, being euthanized or being retired. The P-values derived from log-likelihood ratio tests of univariable logistic regression models examining the association between potential explanatory variables and the. The listed explanatory variables were not included in multivariable analysis as they had $P$-values larger than 0.2 . Data is from 1360 observations of 589 dogs, of which 81 observations were followed by a dog dying or being retired. All dogs were enrolled in the TeamMate project and all observations had recorded values for all tested variables.

\begin{tabular}{ccccc}
\hline & & \multicolumn{2}{c}{ Number of observations } & \\
\cline { 3 - 4 } Variable name & Variable levels & Working & Died or retired & P(LRT) \\
\hline \multirow{2}{*}{ Sex } & Female & 590 & 40 & \multirow{2}{*}{0.57} \\
& Male & 689 & 41 & \\
Body weight & $21 \mathrm{~kg}$ and below & 349 & 22 & \\
(quartiles) & 21.1 to $25 \mathrm{~kg}$ & 311 & 21 & \multirow{2}{*}{0.68} \\
& 25.1 to $30 \mathrm{~kg}$ & 338 & 17 & \\
\hline \multirow{3}{*}{ Body condition score } & $30.1 \mathrm{~kg}$ and above & 281 & 21 & \multirow{2}{*}{0.21} \\
& 1 to 3 & 453 & 35 & \\
\hline \multirow{2}{*}{ Skin abnormalities } & 4 to 5 & 953 & 61 & \multirow{2}{*}{0.48} \\
& 6 to 9 & 99 & 11 & \\
\hline \multirow{2}{*}{ Types of terrain } & No & 691 & 47 & \\
& Yes & 588 & 34 & \\
& Flat and steep & 694 & 47 & \\
\hline
\end{tabular}


Table 8: The results of univariable logistic regression models examining the risk of each visit being followed by dogs dying or being retired in relation to a range of explanatory variables. $\beta$ coefficients (with standard errors (SE)) and odds ratios (with 95\% CIs) derived from the logistic regression models and P-values derived from log-likelihood ratio tests. Explanatory variables with $\mathrm{P}<0.2$ are reported. Data is from 1360 observations of 589 dogs, of which 81 observations were followed by a dog dying or being retired. All dogs were enrolled in the TeamMate project and all observations had recorded values for all tested variables.

\begin{tabular}{|c|c|c|c|c|c|c|c|c|c|c|}
\hline \multirow[b]{2}{*}{ Explanatory variables } & \multirow[b]{2}{*}{ Level } & \multicolumn{4}{|c|}{ Number $(\%)$ of observations } & \multirow{2}{*}{\multicolumn{2}{|c|}{$\beta$ coefficient (SE) }} & \multirow{2}{*}{\multicolumn{2}{|c|}{ Odds ratio $(95 \% \mathrm{CI})$}} & \multirow[b]{2}{*}{$P($ LRT $)$} \\
\hline & & \multicolumn{2}{|c|}{ Working } & \multicolumn{2}{|c|}{ Died or retired } & & & & & \\
\hline \multirow{5}{*}{ Age category } & 1.5 to 2.9 years & 275 & $(20)$ & 11 & $(1)$ & Ref & & Ref & & $<0.0001$ \\
\hline & 3 to 4.9 years & 402 & $(30)$ & 6 & $(0)$ & -1.0 & $(-1.5--0.5)$ & 0.4 & $(0.1-1.0)$ & \\
\hline & 5 to 6.9 years & 260 & $(19)$ & 11 & $(1)$ & 0.1 & $(-0.4-0.5)$ & 1.1 & $(0.5-2.5)$ & \\
\hline & 7 to 9.9 years & 265 & (19) & 22 & $(2)$ & 0.7 & $(0.4-1.1)$ & 2.1 & $(1.0-4.4)$ & \\
\hline & 10 years and older & 77 & $(6)$ & 31 & $(2)$ & 2.3 & $(1.9-2.7)$ & 10.1 & $(4.8-20.9)$ & \\
\hline Number of recorded abnormalities & (count) & \multicolumn{2}{|c|}{-} & & & 0.2 & $(0.1-0.2)$ & 1.2 & $(1.1-1.3)$ & $<0.0001$ \\
\hline \multirow{2}{*}{ Eye abnormalities } & No & 1188 & $(87)$ & 62 & (5) & Ref & & Ref & & $<0.0001$ \\
\hline & Yes & 91 & $(7)$ & 19 & $(1)$ & 1.4 & $(1.1-1.7)$ & 4.0 & $(2.3-7.0)$ & \\
\hline \multirow{2}{*}{ Mouth and teeth abnormalities } & No & 764 & $(56)$ & 32 & $(2)$ & Ref & & Ref & & 0.0004 \\
\hline & Yes & 515 & $(38)$ & 49 & $(4)$ & 0.8 & $(0.6-1.1)$ & 2.3 & $(1.4-3.6)$ & \\
\hline \multirow{2}{*}{ Lameness on trot } & No & 1123 & (83) & 59 & (4) & Ref & & Ref & & 0.0005 \\
\hline & Yes & 156 & $(11)$ & 22 & $(2)$ & 1.0 & $(0.7-1.3)$ & 2.7 & $(1.6-4.5)$ & \\
\hline \multirow{2}{*}{ Reproductive system abnormalities } & No & 1194 & $(88)$ & 68 & $(5)$ & Ref & & Ref & & 0.005 \\
\hline & Yes & 85 & $(6)$ & 13 & $(1)$ & 1.0 & $(0.7-1.3)$ & 2.7 & $(1.4-5.1)$ & \\
\hline \multirow{2}{*}{ Musculoskeletal abnormalities } & No & 674 & $(50)$ & 28 & $(2)$ & Ref & & Ref & & 0.001 \\
\hline & Yes & 60 & $(44)$ & 53 & $(4)$ & 0.7 & $(0.5-1.0)$ & 2.1 & $(1.3-3.4)$ & \\
\hline \multirow{2}{*}{ Neuter status } & Entire & 1189 & $(87)$ & 68 & (5) & Ref & & Ref & & 0.01 \\
\hline & Neutered & 90 & $(7)$ & 13 & $(1)$ & 0.9 & $(0.6-1.2)$ & 2.5 & $(1.3-4.7)$ & \\
\hline Visit number & (count) & & & & & 0.3 & $(0.2-0.4)$ & 1.3 & $(1.1-1.6)$ & 0.01 \\
\hline
\end{tabular}


Table 9: Results of the final multivariable logistic mixed model showing the effect of a range of explanatory variables on the risk of observations being followed by dogs' dying or being retired. Individual dogs and dog owners were defined as nested random effects. Data used in the final model is from 1360 observations of 589 dogs, of which 81 observations were followed by the dog dying, being euthanized or being retired. All dogs were enrolled in the TeamMate project.

\begin{tabular}{|c|c|c|c|c|}
\hline \multirow{2}{*}{$\begin{array}{l}\text { Explanatory variables } \\
\text { Observation number }\end{array}$} & \multirow{2}{*}{$\frac{\text { Level }}{\text { (count) }}$} & \multicolumn{2}{|c|}{ Odds ratio $(95 \% \mathrm{CI})$} & \multirow{2}{*}{$\frac{P(\text { LRT })}{0.15}$} \\
\hline & & 1.2 & $(0.9-1.5)$ & \\
\hline \multirow{5}{*}{ Age category } & 1.5 to 2.9 years & Ref & & $<0.0001$ \\
\hline & 3 to 4.9 years & 0.3 & $(0.1-0.9)$ & \\
\hline & 5 to 6.9 years & 1.0 & $(0.5-2.4)$ & \\
\hline & 7 to 9.9 years & 1.8 & $(0.8-3.8)$ & \\
\hline & 10 years and older & 8.3 & $(3.9-17.7)$ & \\
\hline \multirow{2}{*}{ Lameness on trot } & No & Ref & & 0.03 \\
\hline & Yes & 2.0 & $(1.2-3.4)$ & \\
\hline $\begin{array}{l}\text { Dogs and dog owners } \\
\text { (random effects) }\end{array}$ & & & & 1.0 \\
\hline
\end{tabular}

\section{Discussion}

This is the first time risk factors related to death, euthanasia or retirement have been explored in working farm dogs. Being lame on trot almost doubled the risk of farm dogs being lost from the workforce, suggesting that preventing dogs from becoming lame could reduce their risk of being lost from the workforce significantly. Due to the physical requirements of the work farm dogs do this increase in risk is to be expected, particularly if the lameness is long-lasting and cannot be effectively treated. Lameness can be a sign of musculoskeletal pain and stiffness in dogs and conditions such as cranial cruciate ligament disease and joint dysplasia are common causes of lameness in dogs [18]. However, dogs can also be lame from other causes such as, for example, trauma to the footpads. Because we did not incorporate diagnoses in our data, we do not know what caused the lameness that was recorded in dogs enrolled in this study and musculoskeletal abnormalities were analyzed as a separate risk factor from lameness. When analyzed, the presence of musculoskeletal abnormalities was not found to have a significant effect on the risk of dogs being lost from the workforce despite the significant effect caused by lameness and the likelihood that many cases of lameness were caused by underlying musculoskeletal conditions. One reason for this apparent discrepancy may be the way we analyzed our data. In this study we examined what effect risk factors had in the months immediately following each observation of a dog. As musculoskeletal disease often develops over long periods of time before it progresses to cause pain or lameness, it may be that our analysis considered too short periods of time to detect the effect of musculoskeletal abnormalities on working farm dogs. However, it is likely that musculoskeletal injury and disease is the underlying cause of many the recorded cases of lameness in this study. Research into what types of disorders commonly cause lameness in working farm dogs would be helpful. The results of such research may enable dog owners and veterinarians to treat these conditions more effectively and delay or prevent dogs from developing lameness that could cause them to be removed from work.

Another possible reason for the apparent discrepancy between the effects of lameness and musculoskeletal abnormalities on dogs being lost from work could also be that dog owners are more able to recognize lameness in their dogs than more subtle signs of musculoskeletal disease. Decisions about whether to euthanize or retire working farm dogs are made by their owners, presumably based on their needs on farm and their perception of the performance, health and welfare of their dogs. Many of the most common types of musculoskeletal abnormalities recorded for TeamMate, such as reduced range of joint 
motion, crepitus and joint pain [4,5], are likely to be difficult for dog owners to detect. Changes in movement, behavior and performance in working farm dogs are probably easier for dog owners to notice than subtle musculoskeletal changes, especially when they know their dogs intimately and rely on them to be able to work. As such, musculoskeletal abnormalities are unlikely to affect owners' decisions on whether to remove dogs from work until they are serious enough to cause dogs to become lame. Lameness and related problems with joint stiffness or pain can cause dogs to have difficulties with, for example, jumping up and down from vehicles or across fences, and to have reduced working performance [19]. A study of military working dogs found that dogs were more likely to have signs of spinal disease if they were reported by their handlers to have developed problematic behaviors such as reluctance to jump up onto objects or vehicles, reluctance to perform work tasks or to have become aggressive or anxious [20]. If musculoskeletal abnormalities can be detected and treated early enough, the development of irreversible disease can sometimes be slowed down [21]. Providing farm dog owners with the necessary skills to detect musculoskeletal abnormalities before they progress to cause lameness could enable them to seek veterinary treatment early enough to prevent more serious injury or disease from developing. For example, farm dog owners could be trained in how to detect subtle changes in joint motion or signs of pain in their dogs and to recognize changes in behavior and performance that may indicate pain or discomfort. Helping farm dog owners to recognize early signs of musculoskeletal disease in their dogs could help them to make informed decisions around treatment, retirement and euthanasia.

Age group and the presence of lameness had the strongest effects on the risk of death, euthanasia or retirement in working farm dogs (Table 9). However, acute injury or illness was the most commonly reported reason for dogs being lost from the workforce (Table 4). The high proportion of dogs reported as being lost due to acute injuries or illnesses can be linked to our analysis on risk factors. Dogs that are lame or are suffering from agerelated reduction in body function are probably less able to cope with the physical demands of their work, putting them at increased risk of serious acute injuries that can cause them to be retired or euthanized. For example, dogs that are old or lame may plausibly be less able jump over obstacles such as fence lines or avoid being hit by vehicles or stock. Additionally, young dogs have been shown to require veterinary treatment for acute injuries more often than older dogs [22], possibly due to their lower levels of training and higher excitability. Dog owners could counteract the increased risk due to youth or seniority by adjusting dogs' training and workload according to age. Additionally, prevention and effective treatment of the underlying causes of lameness should be a priority for veterinarians and working dog owners. Doing so would not only improve dogs' health and welfare but could additionally prevent dogs from having serious injuries that cause them to be lost from the workforce prematurely. However, there is currently no data on how dog owners currently work to counteract injury and disease in their dogs or whether there are specific areas where improvements to common practices could be made.

Our results indicate that the risk of working farm dogs being lost from the workforce did not increase markedly until they were ten years or older (Table 9). Nearly three quarters of dogs that were lost from the workforce were seven years or older on their last observation and, of retired dogs, three-quarters were ten years or older. Depending on body size, dogs can generally be considered as being senior at six to seven years of age and geriatric at around nine to 11 years [23], meaning that a majority of working farm dogs that were lost from the workforce could be said to have reached old age. For comparison, only $40 \%$ of police working dogs in New Zealand worked until the standard retirement age of eight years, and the median age at which police dogs left the workforce was 6.6 years [24]. Guide dogs in the United Kingdom worked for a mean of 8.5 years, or until they were around 10 or 11 years old [8], and military working dogs had a mean age at death of ten years [10]. Considering the high activity levels and potential for traumatic injuries, it is reasonable to expect that working farm dogs have shorter working lives than other working dogs such as police, military and guide dogs that are normally bred and trained by institutions and closely followed up by veterinarians throughout their lives. 
However, our data indicates that working farm dogs have similar or possibly longer careers than such working dogs.

A possible reason for the long recorded working lifespans of working farm dogs is that it can be difficult to define what retirement means in these farm dogs. Anecdotally, instead of having a clear cut-off point in either age or health status where dogs are removed from work and moved into retirement, farm dog owners make the decision on whether and how much their dogs should work based on their own knowledge and experience. Often this means that instead of being retired, older dogs' workloads are gradually reduced according to their working capacity and performance, the owners' needs, and the composition of the owners' teams of dogs. If the owner has younger dogs that can replace the old dog satisfactorily, old dogs' workloads will probably be reduced. However, fully trained and experienced older farm dogs can be very valuable to farmers, and owners may be reluctant to retire them as long as they are still able to work. Additionally, working farm dogs are highly motivated to work with stock even as they grow older and some may not be suitable to keep as house pets. As such their owners may feel that their welfare would be impaired if they were not allowed to work. Anecdotally, there were cases in TeamMate where older dogs were noted as being allowed to 'tag along' for work, and dogs as old as 14 were enrolled in the study [4]. These dogs are still exposed to risk factors related to work and may be at higher risk of injury due to lower physical capacity caused by aging [23]. Including such semi-retired dogs in our study population may have caused us to underestimate the number of dogs that would be considered as retired by their owners and to overestimate the number of dogs that die or are euthanized while still an active part of the workforce. However, we felt that excluding semi-retired dogs would be difficult to do in practice due to the lack of a clear definition about what constitutes retirement. We therefore chose to define all dogs that were reported to be still working in any capacity as not retired.

Although our findings indicate that working farm dogs may have equally long working lives as police, military and guide dogs, these results should be interpreted with caution. Due to differences in data collection and the statistical methods used to analyze data it is difficult to compare the studies on police, military and guide dogs directly with our results, and any apparent similarities or differences in results may be misleading. Additionally, police, military and guide dogs carry out work that can be crucial to the safety of humans, and as such any decrease in working performance is likely to cause them to be removed from work. In comparison, a working farm dog that is performing poorly due to age or injury may cause its owner to lose time and money, or cause injury to itself or other animals. However, since such farm dogs can still be useful and opportunities often exists for other dogs on the team to take over the most demanding work, or for dogs to be rehomed to less demanding farms, underperforming farm dogs may be less likely to be removed from the workforce than underperforming police, military or guide dogs. If working farm dogs continue working while their performance is lowered due to illness or injury, this may compromise their welfare and prevent them from fully recovering. However, to determine whether husbandry practices can be improved more data is needed on the health and workloads of older working farm dogs, and how farm dog owners make decisions about when to remove dogs from stock work.

The effect of age on dogs' risk of dying or being retired was not linear. Instead, the lowest risk was seen in dogs between three and 4.9 years old, rather than the youngest group of 1.5 to 2.9-year-olds (Table 9). This is similar to the analysis done by Sheard [3], which showed that dogs that were two years old or younger, or older than seven years old, were the most likely to be reported as having died in a 12-month period. Sheard also found that partially trained dogs were more likely to die than those that were fully trained. Due to differences in behavior, fitness and training level, young dogs are likely to be exposed to somewhat different risk factors than older dogs, and they may be at higher risk of traumatic injury due to their lack of experience and higher levels of excitability. In Sweden, a study of insured pet dogs found that young dogs have a higher risk of receiving veterinary care due to traumatic injury than older dogs [22]. Additionally, young and 
partially trained dogs are sometimes unsuitable for stock work in general or incompatible with their current owner. In Australia, stock dog handlers reported that $20 \%$ of acquired dogs failed to become trained working dogs [25]. Of these, $89 \%$ were dismissed due to problems around temperament and training with more than half of dismissed dogs being reported to have a lack of natural working ability. While dogs that are simply incompatible with their owner can be rehomed or sold, finding new homes for farm dogs that are generally unsuitable for work is likely to be difficult due to their high energy levels and need for stimulation. It is therefore likely that a proportion of such dogs are euthanized rather than rehomed or sold.

Although not statistically significant, the observed effect of eye abnormalities on the risk of death, euthanasia or retirement in working farm dogs warrants further investigation. Reduced eyesight is a plausible cause of increased risk of acute injury that can lead to dogs leaving the workforce. However, it is likely that not all eye abnormalities recorded in this study were associated with reduced eyesight. For example, a majority of the recorded abnormalities on enrolment to the study were described as 'lens opacity' [4]. A common cause of lens opacity - especially in older dogs - is lens sclerosis, a condition that is not associated with a reduction in vision [26]. A possible alternative explanation for the effect of eye abnormalities on the risk of dogs being lost from work could be that dog owners are more likely to remove dogs with visible cloudiness in the eyes, irrespective of whether the dog's vision is impaired. As mentioned, such cloudiness is more likely to occur in older dogs and may be a contributing factor to dogs being euthanized or retired due to 'old age'. While our model did adjust for age group, we lost a good deal of detail in our age data by categorizing dogs' ages, and we can therefore not rule out that the effect of eye abnormalities was confounded by dogs' ages. Further investigation should be carried out to confirm or disprove whether there is a significant effect of eye abnormalities on the risk of working farm dogs dying, being euthanized or being retired.

Neither body condition score nor the ratio of predicted lean body mass to skeletal size score were found to be associated with the risk of dogs dying or being retired. This should not be interpreted as a lack of risk associated with being clinically under- or overweight. Instead, our evidence indicates that these representations of body condition are not predictive of whether dogs die or are retired. It may be that one or both measures are associated with welfare, working performance or other types of health outcomes in working farm dogs. However, in the current study there is little evidence to suggest that either measure is a reliable indicator of overall health in lean, athletic dogs. Given that concern has been expressed that working farm dogs may in general be too thin, more investigation into what constitutes optimal body condition in relation to health in working farm dogs may be warranted.

\section{Conclusion}

This study found that age group and lameness had significant effects on the risk of working farm dogs dying or being retired from work, and that a majority of were working with stock into old age. Although more can doubtless be done to investigate and improve the health and welfare of working farm dogs while they are part of the workforce, there is probably little to be gained in attempting to extend dogs' working careers. However, due to a lack of quality in our data we did not examine factors which may affect the risk of developing disease or lameness, or carry out any assessment on the effects of husbandry practices such as feeding or the quality of housing. Future investigations should focus on these issues, as they are important in allowing dog owners to improve the overall health and welfare of their working farm dogs. Additionally, efforts might be made to investigate health and welfare in older working dogs, and how owners make decisions around whether to euthanize or retire dogs whose ability to work is declining. Such investigation has the potential to greatly improve the health and welfare of working farm dogs.

Author Contributions: Conceptualization, N.C. and L.L.; methodology, N.C., L.L. and K.E.I.; formal analysis, K.E.I..; investigation, N.C., K.E.I., L.L and H.H..; resources, L.L. and H.H.; data curation, 
K.E.I.; writing - original draft preparation, K.E.I.; writing-review and editing, K.E.I., N.J.C., E.J.N. and N.C.; visualization, K.E.I.; supervision, N.J.C., E.J.N. and N.C.; project administration, N.C. and H.H.; funding acquisition, N.C and L.L. All authors have read and agreed to the published version of the manuscript.

Funding: Funding was provided by Vetlife in the form of time, resources and staff needed to plan the project and carry out data collection. The Massey University Working Dog Centre, The Massey University Graduate Research School and the Massey University School of Veterinary Sciences provided funding in the form of postgraduate research grants. The Massey University Working Dog Centre contributed NZ\$18000 towards help with data entry. Vetlife received 3-year sponsorships towards the running of TeamMate from Zoetis (NZ\$10000 pr year), Masterpet (NZ\$ 7500 pr year), Virbac (NZ\$ 4000 pr year) and Gribbles Veterinary Pathology (15\% discount on any laboratory tests for dogs enrolled in TeamMate).

Institutional Review Board Statement: This study has been approved by the Massey University Animal Ethics committee (protocols 15/26 and 18/53). All dog owners have given oral consent to their dogs being included in the study. Written consent is not a requirement in New Zealand and there are many cases in which projects will be approved without written consent. In this survey verbal consent was considered both acceptable and appropriate: 1) the dog owners had to agree to allow the veterinarian to visit the property, 2) when the veterinarian arrived the dog owners had to consent to them being there and 3) the owner had to provide the dog to the veterinarian for physical examination. Further, at each round of data collection dog owners were free to withdraw. Several did withdraw from the study and others did not return phone calls. In terms of the actual process of ethical approval, when the proposal was sent to the Massey University Animal Ethics Committee the method of gaining consent was not included and the Committee did not require the inclusion of this prior to approval.

Data Availability Statement: The datasets generated for this study are available on request to the corresponding author.

Acknowledgments: The authors would like to acknowledge all the staff at Vetlife and the working farm dog owners who made this project possible. Also, we would like to thank Dr Ahmed Fayaz for his help in setting up and managing the database that houses the TeamMate data, and Margaret Gater for her help with data entry. Lastly, we want to acknowledge Zoetis, Masterpet, Virbac and Gribbles Veterinary Pathology for their contributions towards the running of the project.

Conflicts of Interest: The authors declare no conflict of interest. The funders had no role in the design of the study; in the collection, analyses, or interpretation of data; in the writing of the manuscript, or in the decision to publish the results.

\section{References}

1. Singh, I.; Tucker, L.A.; Gendall, P.; Rutherfurd-Markwick, K.J.; Cline, J.; Thomas, D.G. Age, breed, sex distribution and nutrition of a population of working farm dogs in New Zealand: Results of a cross-sectional study of members of the New Zealand sheep dog trial association. N. Z. Vet. J. 2011, 59, 133-138.

2. Cave, N.J.; Bridges, J.P.; Cogger, N.; Farman, R.S. A survey of diseases of working farm dogs in New Zealand. N. Z. Vet. J. 2009, 57, 305-312.

3. Sheard, H. Demographics and health of New Zealand working farm dogs: A survey of dogs on sheep and beef farms in New Zealand in 2009., Massey University: Palmerston North, New Zealand, 2014.

4. Isaksen, K.E.; Linney, L.; Williamson, H.; Cave, N.J.; Beausoleil, N.J.; Norman, E.J.; Cogger, N. TeamMate: A longitudinal study of New Zealand working farm dogs. I. Methods, population characteristics and health on enrolment. BMC Vet. Res. 2020, 16, 1-17.

5. Isaksen, K.E.; Linney, L.; Williamson, H.; Cave, N.J.; Norman, E.J.; Cogger, N. TeamMate: A Longitudinal Study of New Zealand Working Farm Dogs. II. Occurrence of Musculoskeletal Abnormalities. Front. Vet. Sci. 2020, 7, 1-9.

6. Arnott, E.R.; Early, J.B.; Wade, C.M.; McGreevy, P.D. Estimating the economic value of Australian stock herding dogs. Anim. Welf. 2014, 23, 189-197.

7. Cogger, N.; Sheard, H. Working Farm Dogs. In Livestock Production in New Zealand; Stafford, K., Ed.; Massey University Press: 
Auckland, New Zealand, 2017; pp. 280-297 ISBN 9870994136312.

8. Caron-Lormier, G.; England, G.C.W.; Green, M.J.; Asher, L. Using the incidence and impact of health conditions in guide dogs to investigate healthy ageing in working dogs. Vet. J. 2016, 207, 124-130.

9. Worth, A.J.; Cave, N.J. A veterinary perspective on preventing injuries and other problems that shorten the life of working dogs. Rev. Sci. Tech. 2018, 37, 161-169.

10. Moore, G.E.; Burkman, K.D.; Carter, M.N.; Peterson, M.R. Causes of death or reasons for euthanasia in military working dogs: 927 cases (1993-1996). J. Am. Vet. Med. Assoc. 2001, 219, 209-214.

11. WSAVA Global Nutrition Committee Body condition score - dog; World Small Animal Veterinary Association, 2013;

12. Leung, Y.M.; Cave, N.J.; Hodgson, B.A.S. Creation of a predictive equation to estimate fat-free mass and the ratio of fat-free mass to skeletal size using morphometry in lean working farm dogs. N. Z. Vet. J. 2018, 66, 248-256.

13. Dohoo, I.R.; Martin, W.; Stryhn, H. Veterinary Epidemiologic Research; 2nd ed.; VER Inc: Prince Edward Island, Canada, 2009; ISBN 978-0-919013-60-5.

14. R Core Team R: A language and environment for statistical computing; R Foundation for Statistical Computing: Vienna, Austria, 2020;

15. Fox, J.; Weisberg, S. An R Companion to Applied Regression; 3rd ed.; SAGE Publications, Inc.: Thousand Oaks, CA, United States, 2019;

16. Bates, D.; Mächler, M.; Bolker, B.; Walker, S. Fitting Linear Mixed-Effects Models Using lme4. J. Stat. Softw. 2015, 67.

17. Robin, X.; Turck, N.; Hainard, A.; Tiberti, N.; Lisacek, F.; Sanchez, J.-C.; Müller, M. pROC: an open-source package for R and S+ to analyze and compare ROC curves. BMC Bioinformatics 2011, 12, 77.

18. Anderson, K.L.; Zulch, H.; O’Neill, D.G.; Meeson, R.L.; Collins, L.M. Risk factors for canine osteoarthritis and its predisposing arthropathies: A systematic review. Front. Vet. Sci. 2020, 7, 1-16.

19. Mills, D.S.; Demontigny-Bédard, I.; Gruen, M.; Klinck, M.P.; McPeake, K.J.; Barcelos, A.M.; Hewison, L.; Van Haevermaet, H.; Denenberg, S.; Hauser, H.; et al. Pain and problem behavior in cats and dogs. Animals 2020, 10, 1-20.

20. Dodd, T.; Jones, J.C.; Holásková, I.; Mukherjee, M. Behavioral problems may be associated with multilevel lumbosacral stenosis in military working dogs. J. Vet. Behav. 2020, 35, 8-13.

21. Rychel, J.K. Diagnosis and Treatment of Osteoarthritis. Top. Companion Anim. Med. 2010, $25,20-25$.

22. Bonnett, B.N.; Egenvall, A. Age Patterns of Disease and Death in Insured Swedish Dogs, Cats and Horses. J. Comp. Pathol. 2010, 142.

23. Bellows, J.; Colitz, C.M.H.; Daristotle, L.; Ingram, D.K.; Lepine, A.; Marks, S.L.; Sanderson, S.L.; Tomlinson, J.; Zhang, J. Common physical and functional changes associated with aging in dogs. J. Am. Vet. Med. Assoc. 2015, 1, 67-75.

24. Worth, A.J.; Sandford, M.; Gibson, B.; Stratton, R.; Erceg, V.; Bridges, J.P.; Jones, B.R. Causes of loss or retirement from active duty for New Zealand police German shepherd dogs. Anim. Welf. 2013, 22, 166-173.

25. Arnott, E.R.; Early, J.B.; Wade, C.M.; McGreevy, P.D. Environmental factors associated with success rates of Australian stock herding dogs. PLoS One 2014, 9.

26. Davidson, M.G.; Nelms, S.R. Diseases of the Lens and Cataract Formation. In Veterinary Ophthalmology; Gelatt, K.N., Gilger, B.C., Kern, T.J., Eds.; John Wiley \& Sons, Inc.: Ames, Iowa, United States, 2013; pp. 1199-1233. 